\title{
An EM Fault Injection Susceptibility Criterion and its application to the localisation of hotstpots
}

\author{
M. MADAU, M. AGOYAN, P. MAURINE
}

Laboratoire d'Informatique de Robotique et de Microélectronique de Montpellier (LIRMM), STMicroelectronics

2017
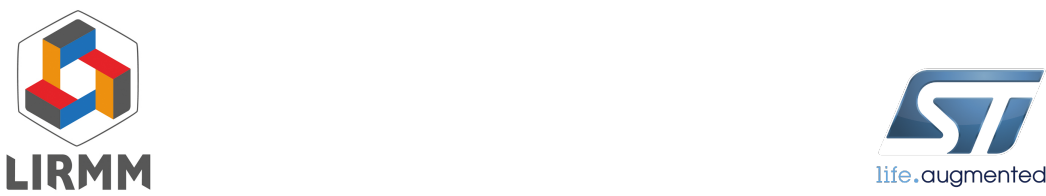


\section{Linking injection/observation channel}

\begin{tabular}{|c|c|l|}
\hline "Attack" & Channel & information from observation channel. \\
\hline $\begin{array}{c}\text { Power } \\
\text { Glitch }\end{array}$ & $V_{d d}$ network & temporal information. \\
\hline $\begin{array}{c}\text { Body Bias } \\
\text { Injection }\end{array}$ & bulk & none. \\
\hline EMFI & EM & temporal and spatial information \\
\hline Laser & photon & $\begin{array}{l}\text { spatial information. } \\
\text { Light observation is expensive. }\end{array}$ \\
\hline
\end{tabular}




\section{Why binding EM analysis to injection?}

EMFI combinatory complexity:

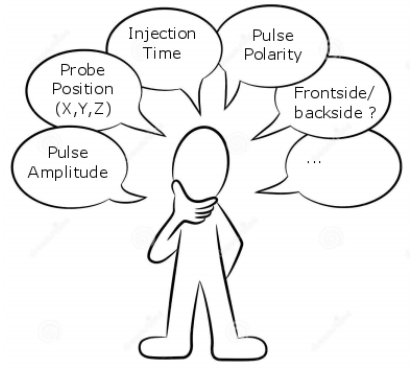

Time efficiency ${ }^{a}$ :

Analysis map $\rightarrow$ one day for three executables.

Injection map (fixed parameters) $\rightarrow$ three days for one executable.

${ }^{a}$ timing are relative to our setup

\section{Aim:}

Ease and fasten EMFI security characterisation $\rightarrow(X, Y)$ position. 


\section{Table of Contents}

Criterion principles

EMFI hotspots definition

Designing the criterion 


\section{EM coupling}

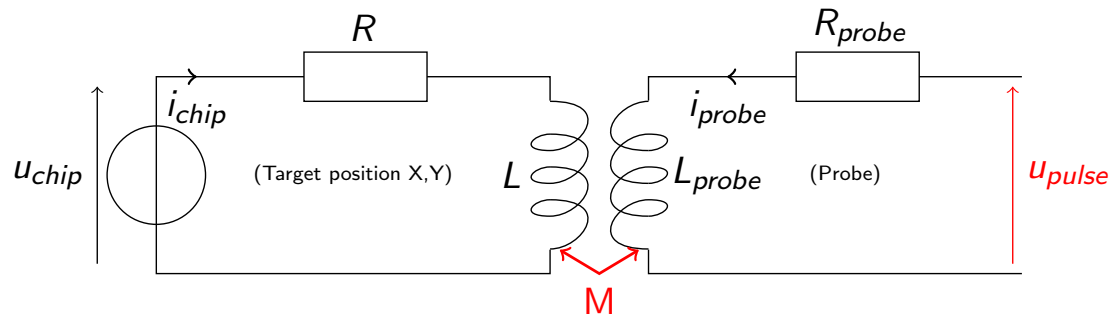

\section{Coupling: (injection case)}

$$
u_{\text {chip }}=R i_{\text {chip }}+L \frac{\mathrm{d} i_{\text {chip }}}{\mathrm{d} t}+M \frac{\mathrm{d} i_{\text {probe }}}{\mathrm{d} t}
$$




\section{EM coupling}

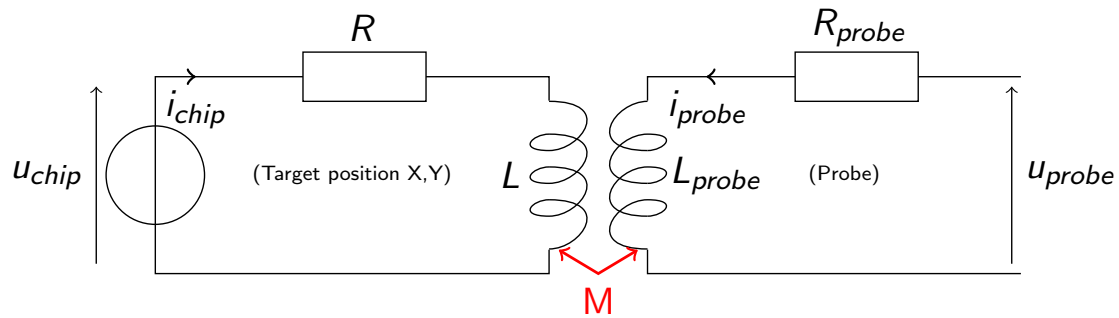

\section{Coupling: (analysis case)}

$$
u_{\text {probe }}=R i_{\text {probe }}+L_{\text {probe }} \frac{\mathrm{d} i_{\text {probe }}}{\mathrm{d} t}+M \frac{\mathrm{d} i_{\text {chip }}}{\mathrm{d} t}
$$




\section{Antenna reciprocity}

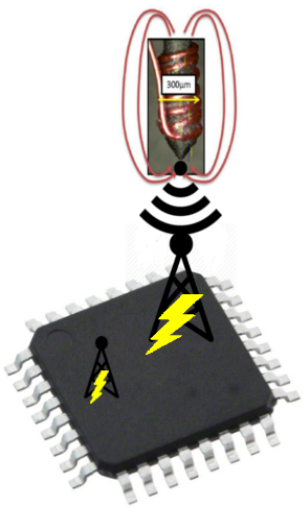

\section{Antenna reciprocity:}

The efficiency of a receiving antenna is as important as its transmitting efficiency.

Conclusion 1:

Finding high emission antenna

$\rightarrow$ best coupling positions on circuits.

Conclusion 2:

High emission antenna $\neq$ best entry point

$\rightarrow$ not necessarily linked to data. 


\section{Sampling fault model ${ }^{1}$}
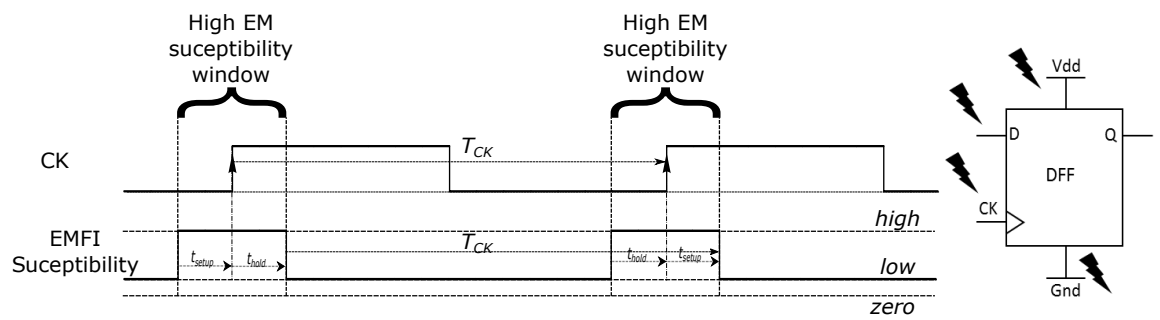

\section{System target:}

$\hookrightarrow$ DFF are more likely to be faulted by EM injection.

$\hookrightarrow$ Target event occurring at $f_{C K}$

${ }^{1}$ EM injection: fault model and locality S. Ordas, L.Guillaume-Sage, P. Maurinne FDTC 2015. 


\section{EMFI Criterion definition}

Area to target are positions:

- (guideline 1) emitting the strongest signal (in terms of power) associated to the clock signal or clock tree.

$\rightarrow$ tool: Power Spectral Density $P S D\left(f_{C K}\right)$

- (guideline 2) emitting signal tightly bind to both targeted algorithm and clock frequency $\left(f_{C K}\right)$.

$\rightarrow$ tool: incoherence $\left(f_{C K}\right)$ 


\section{Guideline 2 tools:}

$$
\operatorname{inc}_{s_{1}, s_{2}}(f)=1-\frac{p s d_{s_{1}, s_{2}}(f)^{2}}{p s d_{s_{1}, s_{1}}(f) \cdot p s d_{s_{2}, s_{2}}(f)}
$$

Notation:

$s_{1}=$ EM emission for input 1 .

$s_{2}=$ EM emission for input 2 .

Aim

$\hookrightarrow$ Look for differences in spectrum occurring at $f_{C K}$ ie DFF used by algorithm. 


\section{How to combine and weight those two measures?}
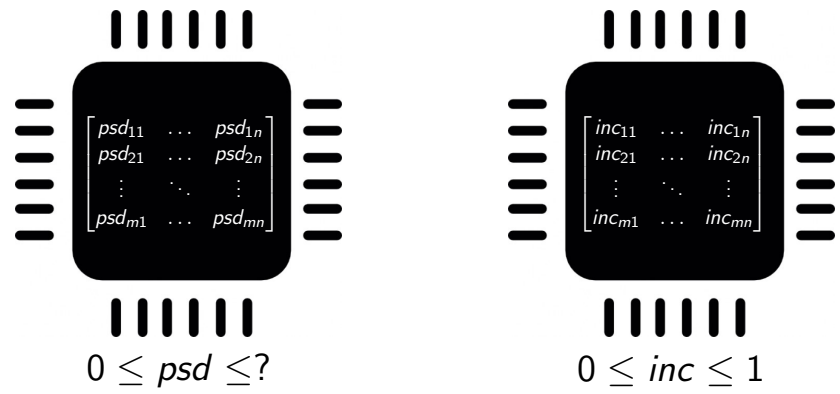


\section{Raw data: PSD, Incoherence view}

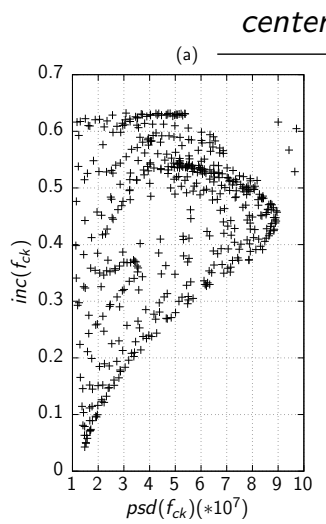

reduce

remaping
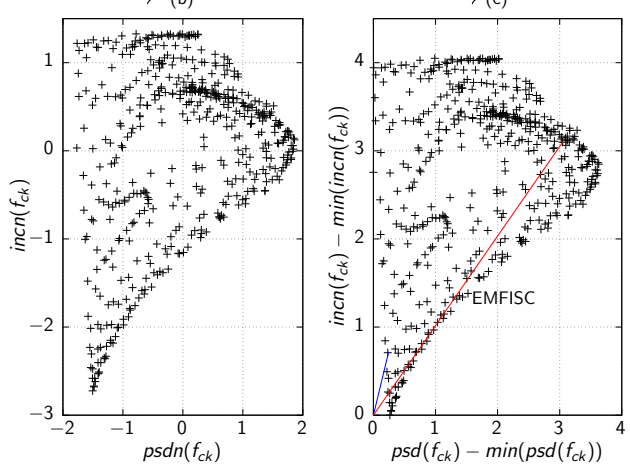

$$
\operatorname{inc}_{s_{1}, s_{2}}(f)=1-\frac{p s d_{s_{1}, s_{2}}(f)^{2}}{p s d_{s_{1}, s_{1}}(f) \cdot p s d_{s_{2}, s_{2}}(f)}
$$




\section{EMFISC Procedure}

\section{Algorithm 1 EMFISC}

Input: $f_{C K}$, matrix of $s_{1}$ and $s_{2}$,

$\alpha$ (\% chip to keep),

a (weight psd compared to incoherence)

Output: emfisc $_{X, y}$

1: for $X, Y$ positions do

2: $\quad$ compute $p s d_{s_{1}}(f)$

3: $\quad$ compute inc $c_{s_{1}, s_{2}}(f)$

4: end for

5: $p s d n_{x, y}$ and $i n c n_{x, y}=$ center reduce $p s d_{x, y}$ and $i n c_{x, y}$ population

6: remap $p s d n_{x, y}$ and $i n c n_{x, y}$ population

7: compute emfisc $c_{x, y}=\sqrt{(1-a) * p s d n_{x, y}^{2}+a * i n c n_{x, y}^{2}}$

LIRMM 8: quantile $\left(\right.$ emfisc $\left._{x, y}, \alpha\right)$ 


\section{Table of Contents}

\section{Criterion principles \\ EMFI hotspots definition \\ Designing the criterion}

Results

\section{Conclusion}

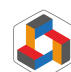

\section{LIRMM}

$\sum_{\text {life.augmented }}$ 


\section{Experimental protocol}

Target algorithm:

Algorithm 2 Pattern (AddrSRAM32, AddrSRAM96)

1: $\mathrm{PUSH}\{\mathrm{Ir}\}$

2: ADD R0,R0,\#0; 11 times

3: LDR R2,[R0]; read SRAM32

4: STR R2,[R1]; write SRAM96

5: LDR R3,[R1]; read back

6: ADD R0,R0,\#0; 11 times

7: $\mathrm{POP}\{\mathrm{pc}\}$ 


\section{EMFISC figures of merit (target 1 130V)}
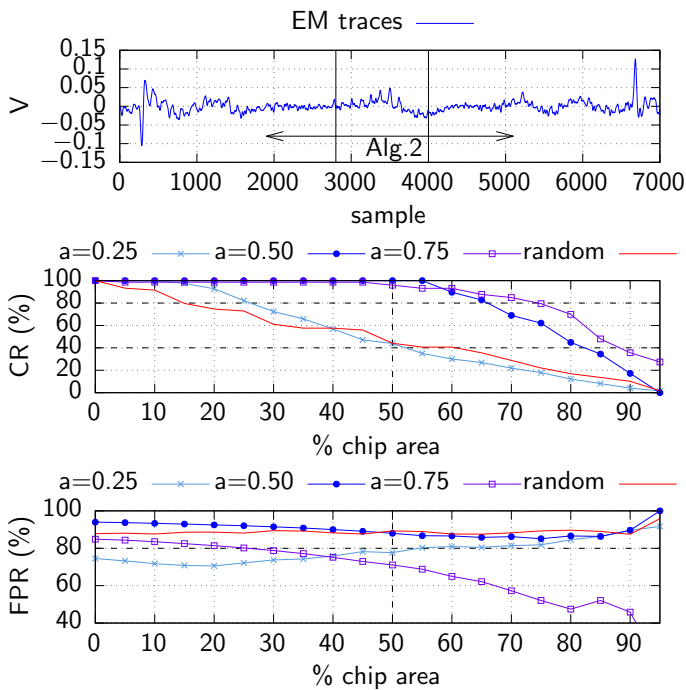


\section{EMFISC figures of merit (target 2 198V)}
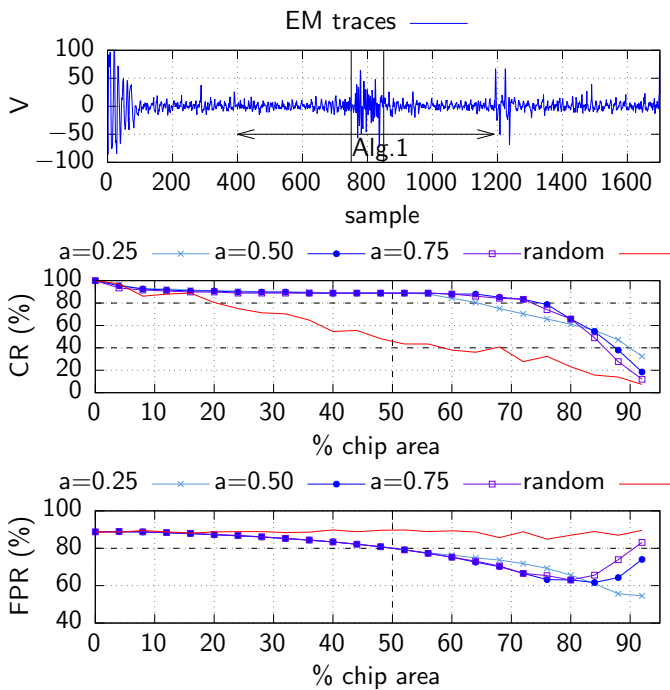


\section{Quantile fixed at 60\% target 1}
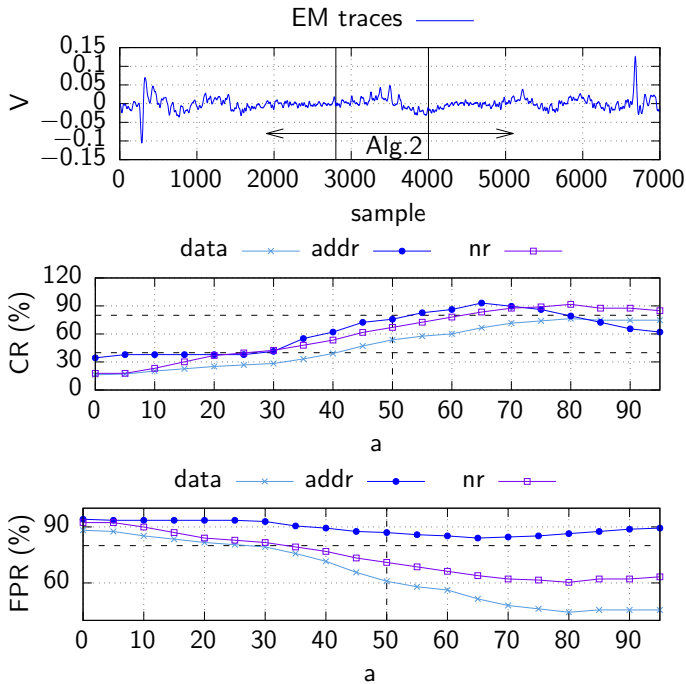


\section{Results:}

- There is a link between EM emissions and EMFI.

- This link can be use to ease EMFI characterisation.

Refining the criterion:

- Other combination of PSD and Incoherence curves.

- Finding a way to weight PSD and Incoherence.

- Adding a criterion more target specific, such as a better measurement of $\mathrm{M}$ parameter. 
Thanks

Any questions?
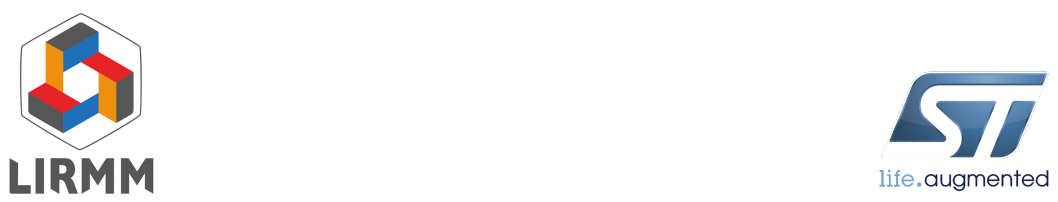